\title{
STRIATAL TYROSINE HYDROXYLASE ACTIVITY: MULTIPLE CONFORMATIONAL KINETIC OSCILLATORS AND PRODUCT CONCENTRATION FREQUENCIES ${ }^{1}$
}

\author{
ARNOLD J. MANDELL AND PATRICK V. RUSSO
}

Department of Psychiatry, University of California, San Diego, La Jolla, California 92093

\begin{abstract}
Samples from rat striate cortices, taken in triplicate at 2-min intervals from a supernatant maintained at $4^{\circ} \mathrm{C}$, then assayed for $1 \mathrm{~min}$ at $37^{\circ} \mathrm{C}$ for tyrosine hydroxylase activity, manifested average amplitudes of time-dependent variation that were related to the level of mean velocity and were not influenced by changing temperature or pharmacological ligands. In contrast, the frequencies of variation manifested characteristic wavelengths of about $26,7.5$, and $5 \mathrm{~min}$, and the relative proportions of those frequencies were changed by an increase in temperature and by certain drugs that are active in the dopamine system (D-amphetamine, haloperidol, and propranolol). Hypothetical, contemporaneous multiple quasi-stable states, each with a characteristic spectral frequency of fluctuation, are consistent with the appearance of reiterative enzyme saturation functions when supernatant and column-enriched preparations were examined within the physiological cofactor range over increasing substrate concentrations. Pharmacologically sensitive time distributions of multiple enzyme conformations, each a kinetic oscillator, determining product concentration frequencies, may be a mechanism by which drugs influence neurotransmitter periodicities in brain.
\end{abstract}

Evidence for conformational heterogeneity and interconvertibility among kinetic forms of brain tyrosine 3 hydroxylase (TOH, EC 1.14.16.2) due to the influence of a wide variety of ligands and conditions has been reviewed elsewhere (Mandell, 1978). In addition, preliminary studies of the time- and/or substrate-dependent kinetics of striatal TOH activity at physiological cofactor concentrations (Bullard et al., 1978; Mandell et al., 1980) revealed multiple characteristic power spectral (frequency) peaks in time and multiple "intermediary saturation plateaus" (Teipel and Koshland, 1969) over substrate, both consistent with the existence of multiple contemporaneous stable states of the enzyme protein (Yellin et al., 1979; Mandell, 1980; Mandell et al., 1981). Now we report a systematic study of the kinetic variations of rat striatal $\mathrm{TOH}$ activity in supernatant and column-enriched preparations over time and over substrate as well as the influences of dopamine-related pharmacological ligands upon them. Our hypothesis is that drug-induced alterations in conformational stability leading to changes in the relative time distributions of multiple enzyme forms (kinetic oscillators) may underlie the varying patterns of product concentration frequencies.

\footnotetext{
${ }^{1}$ This research is supported by United States Public Health Service Grant DA-00265-08. We thank Al P. Turken for excellent technical assistance.
}

\section{Materials and Methods}

We used adult male Sprague-Dawley rats weighing 200 to 300 gm each (Hilltop Laboratories, Scottsdale PA). All compounds and drugs were reagent grade or the equivalent. Isotopically labeled substrate L-3,5-ditritiotyrosine (New England Nuclear Corp., Boston, MA) was purified by ion exchange and lyophilization in the presence of 3 N HCl. Biopterin was obtained from Regis Chemical Co., Morton Grove, IL. Haldol brand of haloperidol was obtained from McNcil Laboratory, Fort Washington, PA; D-amphetamine was obtained from Sigma Chemical Co., St. Louis, MO; and Inderal brand of propranolol hydrochloride was obtained from Ayerst Laboratories, New York, NY. ACS (Amersham/Searle, Arlington Heights, IL) served as the scintillation fluid for the estimation of tritium levels in a Beckman LS-250 liquid scintillation system. Tetrahydrobiopterin $\left(\mathrm{BH}_{4}\right)$ was prepared fresh as needed by catalytic hydrogenation of biopterin $(0.83$ $\mathrm{mg}, 3.5 \mathrm{~mol}$ ) which was suspended in $5 \mathrm{ml}$ of $20 \mathrm{mM} \mathrm{HCl}$. After the addition of $5 \mathrm{mg}$ of $\mathrm{PtO}_{2}$, the sample was shaken and bubbled with hydrogen for $45 \mathrm{~min}$. Platinum was removed by filtration through a $0.45-\mu \mathrm{m}$ Swinnex-13 unit (Millipore Filter Corp., Bedford, MA).

All brain dissections were performed immediately after decapitation on a metal stage maintained at $0^{\circ} \mathrm{C}$ on ice. The hemispheres were separated, and the striate cortices, containing the putamen, caudate nucleus, and some glo- 
bus pallidus and weighing $100 \pm 14 \mathrm{mg}$, were pinched free as described in an earlier article (Geyer et al., 1976). For the characteristic experiment, striatal tissue from 13 brains was pooled and homogenized in 20 parts, w/v, of $2 \mathrm{~mm}$ potassium phosphate buffer $(\mathrm{pH} 7)$ using a glassTeflon homogenizer with a clearance of $0.01 \mathrm{~cm}$. After centrifugation at $35,000 \times g$ for $20 \mathrm{~min}$, the supernatant fraction was kept at $4^{\circ} \mathrm{C}$ on ice for sequential removal of aliquots to be assayed for TOH activity by the method of Nagatsu et al. (1964), as modified in our laboratory (Knapp et al., 1975), in which activity is measured by release of tritiated water from 3,5-ditritiotyrosine. Protein was determined according to the method of Lowry et al. (1951).

The final incubation mixtures of $0.35 \mathrm{ml}$ contained 143 $\mathrm{mm}$ potassium succinate ( $\mathrm{pH} 5.85) ; 17.4 \mathrm{~mm} \beta$-mercaptoethanol; 3 to $50 \mu \mathrm{M}$ tetrahydrobiopterin $\left(\mathrm{BH}_{4}\right) ; 0.15 \mathrm{~mm}$ ferrous sulfate; 8 to $60 \mu \mathrm{M}$ tyrosine, including $0.2 \mu \mathrm{M} 3,5-$ ditritiotyrosine (final specific activity, $0.5 \mu \mathrm{Ci} / \mu \mathrm{mol}$ ); and $100 \mu \mathrm{l}$ of tissue sample.

Routine experiments directed toward time dependence involved 40 triplicate determinations: Over $80 \mathrm{~min}$, three $100-\mu \mathrm{l}$ aliquots were removed from an iced supernatant preparation at 2 -min intervals, added to the reaction mixture, and incubated for $1 \mathrm{~min}$ at $37^{\circ} \mathrm{C}$ for assay of $\mathrm{TOH}$ activity. Triplicate blanks were taken at 0 and 82 min. In replicate experiments, addition of reactants and sample placement in the tube racks were randomized to preclude any spuriously regular frequencies due to routine technical artifact. In no instance in this data set was procedural order related to spectral findings. When drugs were used, they were added to the supernatant source, not to the assay mixtures.

Substrate dependence was examined with determinations of enzyme activity using the assay as described above with $3 \mu \mathrm{M} \mathrm{BH}{ }_{4}$ for 20 min over a range of tyrosine in increments of $5,2,1$, and $0.2 \mu \mathrm{M}$, yielding functions of 15 to 100 points, with the median points of triplicate determinations plotted.

Our raw data are the median points of the triplicate determinations in picomoles per $\mathrm{mg}$ of protein per min. The range of the triplicate determinations, the procedural variance, averaged from 25 to $40 \%$ of the experimental variance, making the signal-to-noise ratio of the spectral information vary from 3 to 1.5 to 1 , well above the threshold of resolution for such approaches (Blackman and Tukey, 1958). For systematic statistical analysis of control and drug-influenced time series, each sequence of values was passed through a linear regression filter (i.e., detrended) so that each original value was transformed into its difference from the mean and normalized with respect to velocity and slope to yield a new time series with a zero mean. The detrended time series were subjected to three kinds of analysis: moments, spectral analysis, and determination of a dimensional exponent. All analysis was done on a Burroughs 7800 computer using routines from the International Mathematics and Statistics Library (IMSL) for power (frequency) spectra, $G x(f)$, and autocorrelation functions, $R x(\tau)$, and programs that we designed to determine the moments (Bendat and Piersol, 1971) and the dimensional exponent, $D$
(Mandelbrot, 1977). In some instances, values for a 40point time series were subjected to the high frequency filtering technique of taking a three-point moving average in which each consecutive point plotted represents the average of itself, the previous, and the following value (Chatfield, 1975).

Because the slope of the linear regression line was characteristically less than 0.005 , its zero time intercept was used to represent the mean velocity of the enzymatic process, $\bar{X}_{0}$ (Fig. 1). In a 40-point detrended time series, where each point is a deviation from $\bar{X}_{0}$ (which now equals 0 ), the root mean square (RMS) value is equivalent to $\sigma$, represents the average amplitude of the fluctuations around the mean, and is also the square root of the variance. In the form of the coefficient of variation, $\sigma / \bar{X}$, the normalized standard deviation is used also to represent the mean amplitude of the variations. Fourier transformation of the time series of deviations from the mean function yields an autocorrelation function, $R x(\tau)$, and Fourier transformation of that function yields a power (frequency) spectrum, $G x(f)$. We restricted the number of lags to 20 and maintained the Nyquist folding frequency at $1 / 4 \mathrm{~min}^{-1}$ which reduced the likelihood of aliasing contributing to the apparent harmonics of the frequencies (Bendat and Piersol, 1971).

In Figure 1, the diagram shows a probability density distribution, that is, the proportion of an ensemble $(y$ axis) occupying each "bin" of a normalized range of values around the zero mean ( $x$ axis) at one point in time. The arrows indicate that the distribution may change over time. The average amplitude of the deviations from the mean is represented by $\sigma$ and $\sigma / X$. The autocorrelation function, reflecting serial correlation, indicates the frequency of variation. For example, if the process is changing quickly (e.g., white noise), serial correlation is

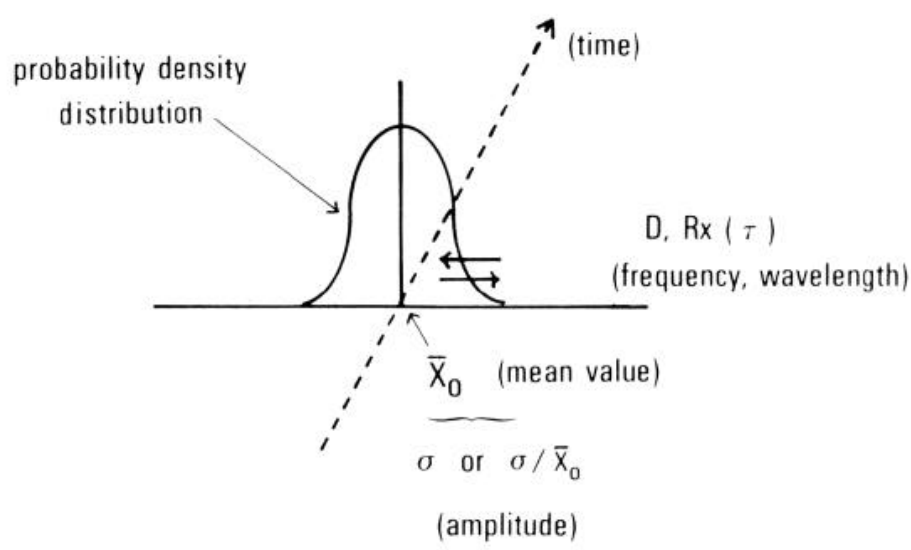

\section{PARAMETERS OF STOCHASTIC PERIODICITY}

Figure 1. Diagram representing a probability density distribution around the mean of an ensemble of determinations in which the ordinate indicates the percentage of the total number of data points at each value along the abscissa. The standard deviation, $\sigma$, or the coefficient of variation, $\sigma / \bar{X}_{0}$, represents the average amplitude of the time-dependent variations. The autocorrelation function, $R x(\tau)$, and the dimensional exponent, $D$, indicate the frequency with which the variations are changing along the time axis. 
low, and the $R x(\tau)$ is smaller. If the variational process is nearly periodic, the oscillating positive and negative values have characteristic wavelengths.

In the analysis of variance by frequency also, that is, in the power spectrum, $G x(f)$, slow and fast periodic variation in the sample distribution function are visible (Bendat and Piersol, 1971). The dimensional exponent, $D$, like $R x(\tau)$-and $G x(f)$, not shown-also indicates the rate of change over time in the variations of the deviations from the mean, reflecting the degree of irregularity of the stochastic surface of the time series (Mandelbrot, 1977).

The dimensional exponent, $D$ (Mandelbrot, 1977), is equal to $1-m$ (see Fig. 2), where $m$ is the negative slope of the straight line generated by linear regression analysis of points produced when the $\log _{10}$ of several intervals of the independent variable (in our experiments, those are minutes of the $0^{\circ} \mathrm{C}$ time course, i.e., $2,4,6,8,10$, etc.) are plotted along the $x$ axis against $\log _{10}$ of points, each of which represents the perimeter of the function and results from summing repeated applications of the distance formula $\sqrt{\left(x-x^{\prime}\right)^{2}+\left(y-y^{\prime}\right)^{2}}$ to the "coastline" generated by each measurement interval. In Figure 2, process $A$ is changing more rapidly; it manifests short, periodic $R x(\tau)$ wavelengths, a more negative slope (higher $m$ ), a more complex surface, i.e., a longer coastline, and a higher $D$. Process $B$, on the other hand, manifests longer periods in $R x(\tau)$, a less negative slope (lower $m$ ), and a lower $D$, all reflecting a more slowly changing process. In more than 200 time series on five enzyme and two receptor binding preparations, we have generally found changes in $D$ of 0.04 to 0.06 to be replicable (Mandell, $1980,1981)$. Overall, $D$ has been both more sensitive and more stable than either $R x(\tau)$ or $G x(f)$, and the computer program for its determination is available on request.

Using comparable spectral approaches to membrane conductance variations, Stevens (1977) suggested that an increase in the reliability of $G x(f)$ could be achieved by averaging. Control and drug effects reported in Table I represent the average power by frequency of eight spectra. Average variation in replicate determinations for
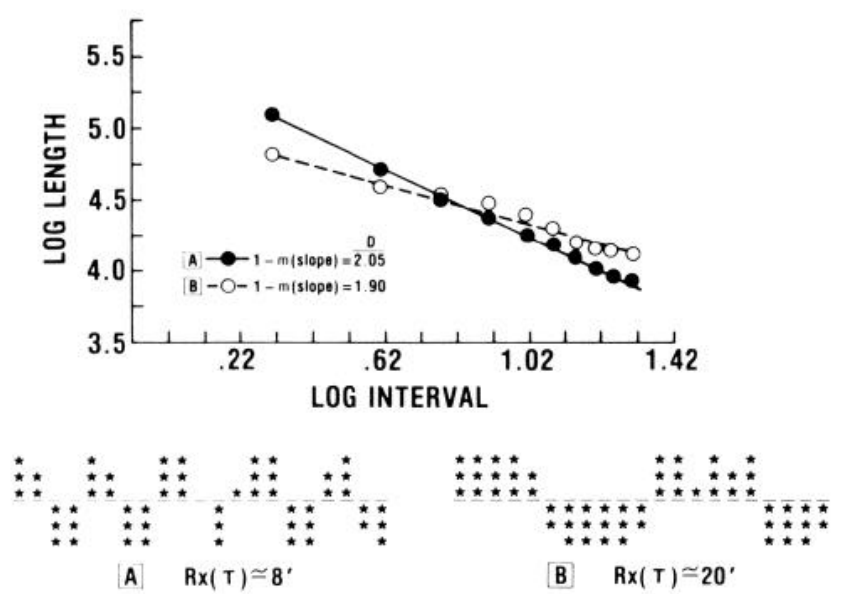

Figure 2. Two indices of variational frequency, the autocorrelation length, $R x(\tau)$, and the dimensional exponent, $D$, in quickly $(A)$ and slowly $(B)$ changing periodic processes. See text. each sample point ranged from 25 to $40 \%$ of the experimental variance while maintaining stable average periodicities over the time series as indicated by averaged $G x(f), R x(\tau)$, and $D$.

In some instances, we used column chromatography to obtain enriched preparations of three molecular weight forms of TOH free of quinonoid dihydropteridine reductase (QDPR) for use in substrate saturation functions (Musacchio et al., 1973; Kuczenski, 1973). Striatal tissue from 30 rats was homogenized in 3 vol of $25 \mathrm{~mm}$ Tris/ acetate buffer ( $\mathrm{pH} 6.0$ ) containing $50 \mathrm{~mm} \mathrm{NaCl}, 50 \mathrm{~mm}$ $\mathrm{KCl}, 0.1 \mathrm{~mm} \mathrm{CaCl}_{2}$, and $0.1 \mathrm{~mm} \mathrm{MgCl}_{2}$, then centrifuged at $35,000 \times g$ for $20 \mathrm{~min}$. The supernatant was applied to a $2.5 \times 90 \mathrm{~cm}$ column packed with Ultrogel ACA 34 polyacrylamide/agarose polymer (LKB), exclusion limits $M_{\mathrm{r}}=20,000$ and 350,000 , which was pre-equilibrated with homogenization buffer. The column was pumped peristaltically at a flow rate of $0.5 \mathrm{~cm}^{3}$ per $\mathrm{min}$, and $7.5-\mathrm{cm}^{3}$ fractions were collected at $4^{\circ} \mathrm{C}$ in a cold room. Pilot runs in the presence of $50 \mathrm{~mm}$ 2-mercaptoethanol did not increase the column yield significantly. After dehydration dialysis, specific activity in the column-enriched fractions was increased 4- to 6-fold over that in the crude supernatant preparations. Assays for TOH activity and protein concentration were carried out as indicated above. QDPR activity, reported to have two characteristic molecular weight forms (Webber and Whitely, 1978) was determined to rule out its coupling with $\mathrm{TOH}$ in the columnenriched preparations and was assayed by the method of Nielsen et al. (1969).

\section{Results}

Figure 3 portrays a characteristic sequence of median values of triplicate determinations of $\mathrm{TOH}$ activity in samples removed from the supernatant fraction of striatal homogenate at 2-min intervals and assayed for $1 \mathrm{~min}$, with the cofactor concentration at $3 \mu \mathrm{M}$. These results were filtered statistically by taking a three-point moving average of the data points which removed the higher frequency fluctuations and made the dominant frequency more prominent. This procedure rules out the possibility that the dominant "slow" resulted from the aliasing of a faster frequency that might have been folded in concomitant with the Fourier transformation. The dominant frequency is seen in the spectrum as $1 / f$, a wavelength of $25.3 \mathrm{~min}$, and in the autocorrelation function, $R x(\tau)$, as $20 \mathrm{~min}$. The dimensional exponent, $D$, equaled 1.70 .

The technique of taking a moving average is particularly useful when comparing the effects of two experimental conditions. Figure 4 shows the results of two experiments conducted simultaneously in which supernatant sources kept at $4^{\circ} \mathrm{C}$ and $22^{\circ} \mathrm{C}$ were sampled alternately and assayed with the cofactor at a concentration of $10 \mu \mathrm{M}$. The fraction kept at $22^{\circ} \mathrm{C}$ manifested faster variations in catalytic velocity than the control kept at $4^{\circ} \mathrm{C}$, as seen in the $G x(f)$ and $R x(\tau)$ and reflected in the higher $D$ value. A systematic study of the energy of activation using a range of temperatures is currently under way. Preliminary findings suggest over a 4-fold change in dominant frequency from $0^{\circ} \mathrm{C}$ to $37^{\circ} \mathrm{C}$.

Although a moving average is useful in simplifying the analysis of fluctuations, it may distort the appearance of 

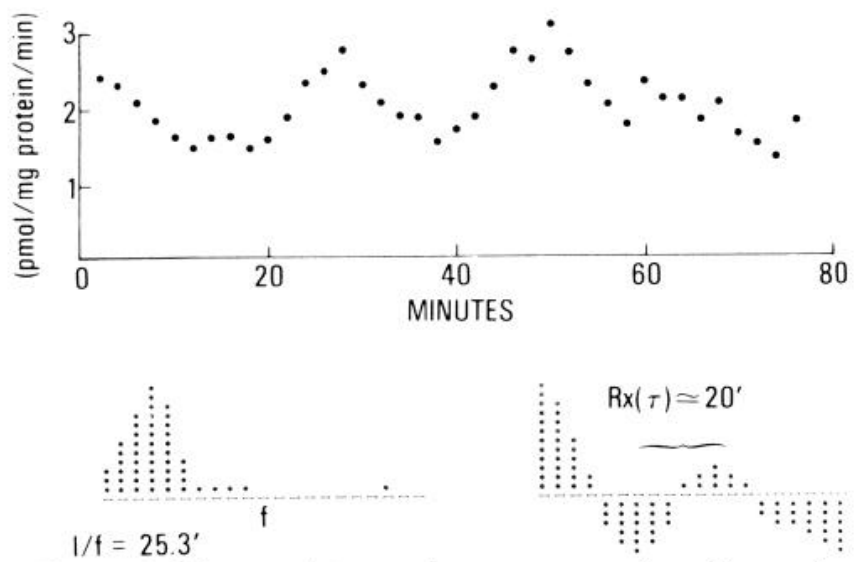

Figure 3. Three-point moving averages of median values from triplicate determinations of TOH activity. Aliquots of 100 $\mu \mathrm{l}$ were removed at 2 -min intervals from a rat striatal supernatant fraction kept at $4^{\circ} \mathrm{C}$ and assayed for catalytic activity at $37^{\circ} \mathrm{C}$ for $1 \mathrm{~min}$ in the presence of $3 \mu \mathrm{M} \mathrm{BH}{ }_{4}$ and $8 \mu \mathrm{M}$ tyrosine. The power (frequency) spectrum, $G x(f)$, demonstrates a dominant wavelength of $25.3 \mathrm{~min}$. The autocorrelation function, $R x(\tau)$, manifests a prominent period of about $20 \mathrm{~min}$. $D$ equaled 1.70 .
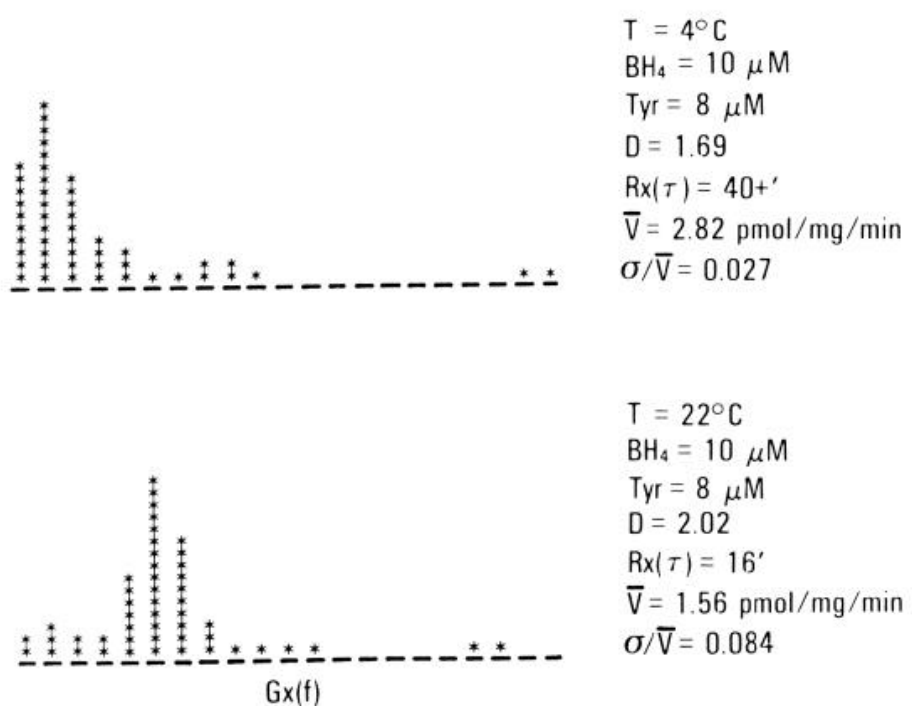

Figure 4. Summary of the results of two simultaneous 40point time courses in triplicate, as in Figure 3 , in which the supernatant sources for the aliquots were kept at $4^{\circ} \mathrm{C}$ and $22^{\circ} \mathrm{C}$, respectively. Assayed with $\mathrm{BH}_{4}$ at $10 \mu \mathrm{M}$, the room temperature series manifested faster frequencies $(G x(f), R x(\tau)$, and $D)$ than that kept at $4^{\circ} \mathrm{C}$. The amplitude of variation changed as might be expected from the alteration in average velocity, $\bar{V}$. That is, a lower $\bar{V}$ was associated with a higher $\sigma / \bar{V}$. See Figures 6 and 7. $T$, temperature; $T y r$, tyrosine.

a stochastic process into one suggesting the behavior of a linearly deterministic oscillator (Slutzky, 1937). Figure 5 shows four representative time series of the sort that we use in the remainder of the analyses reported here, that is, median values of triplicate determinations per se. When the supernatant was kept at $4^{\circ} \mathrm{C}$ and the $\mathrm{TOH}$ activity was assayed with $3 \mu \mathrm{M} \mathrm{BH}_{4}$, spectral analysis of the frequencies of the fluctuations showed peaks indicating wavelengths of 20 to 28,7 to 8 , and 4 to $6 \mathrm{~min}$. Panels
$A$ and $B$ represent control conditions. The higher average level of the process in $A$ is associated with a lower coefficient of variation, $\sigma / \bar{X}$. Panel $C$ represents a time series for which the supernatant fraction was made $1 \mu \mathrm{M}$ in D-amphetamine sulfate as the free base; the spectrum indicates a more symmetrical distribution of power among the three wavelengths. The faster average frequency of variation in the amphetamine-treated supernatant fractions is reflected also in a reduction of $R x(\tau)$ to $6 \mathrm{~min}$ and in an increase in the dimensional exponent, $D$, to 2.05 . Panel $D$ represents a characteristic time series for which the supernatant was made $5 \mu \mathrm{M}$ in propranolol. Regular periodicity of 7 to $8 \mathrm{~min}$ is indicated by the dominant spectral peak with half-maximal power "shoulders" on either side (a Hanning window, Chatfield, 1975) and by the regular oscillations of the velocity plot itself. Both $R x(\tau)$ and $D$ reflect the faster average frequency of fluctuation induced by propranolol compared with control.

The average amplitude of the fluctuations, $\sigma$, varies as a function of the average level of the velocity. In Figure 6 , each of the 12 points represents the average standard deviation from mean velocity obtained by combining results from 12 40-point time courses in which medians of triplicate determinations served as raw data. When mean velocity was above $4 \mathrm{pmol} / \mathrm{mg}$ of protein $/ \mathrm{min}$, the values for $\sigma$ were stable, but as the average catalytic rate fell, $\sigma$ climbed exponentially.

The presence of pharmacological ligands did not influence the slope, as indicated in Figure 7, which is a plot of the $\log _{10}$ of the average velocity versus the $\log _{10}$ of the coefficient of variation. Here the quadratic exponential relationship can be seen in the log-log slope of -2 . Com-
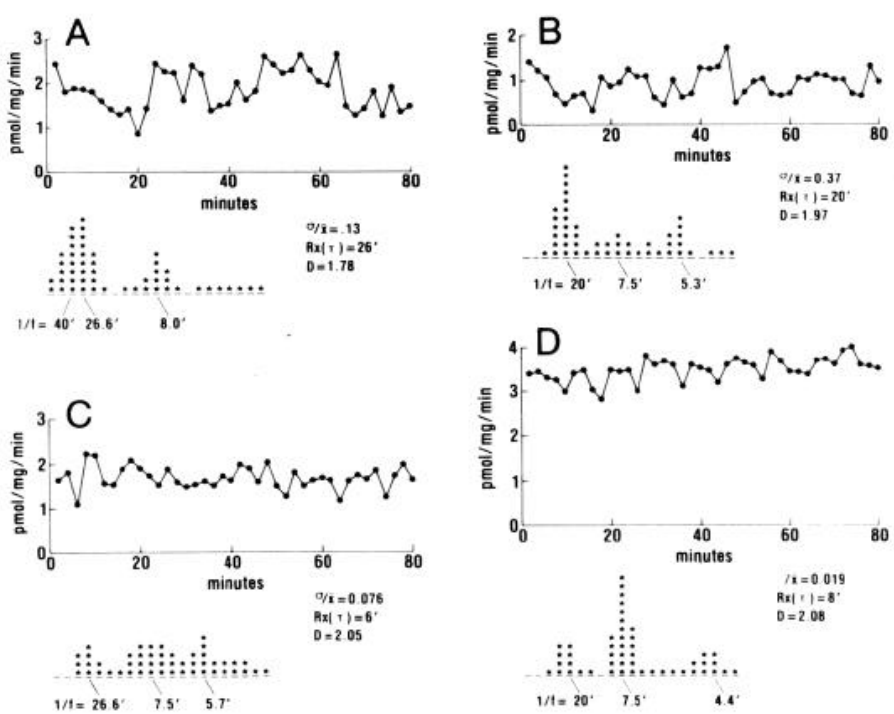

Figure 5. Four representative 80-min time series as in Figures 3 and 4 but without the high frequency filter of the three-point moving average. Panels $A$ and $B$ refer to control experiments in which the lower level of $\bar{V}(B)$ is associated with a higher coefficient of variation, $\sigma / \bar{X}$. Panel $C$ portrays the shift in power to the middle wavelengths and the faster overall frequencies $(R x(\tau)$ and $D)$ characteristic of the effect of $1 \mu \mathrm{M}$ D-amphetamine. Panel $D$ indicates the more regular and faster periods induced by $5 \mu \mathrm{M}$ propranolol. 
TYROSINE HYDROXYLASE ACTIVITY

\section{RELATION BETWEEN AVERAGE VELOCITY AND STANDARD DEVIATION}

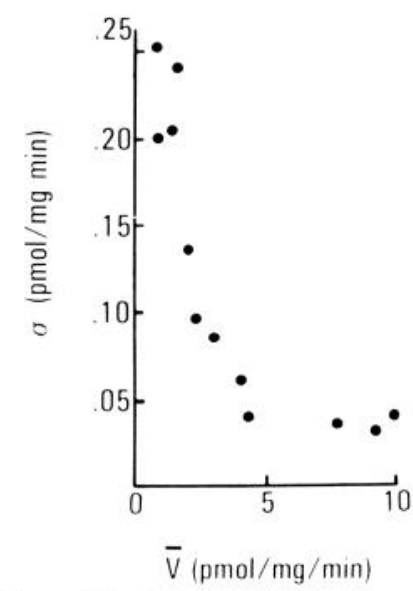

Figure 6. Each point represents the average velocity and standard deviation from one of 12 40-point time series in triplicate, demonstrating the exponential relationship between average amplitude of variation and catalytic velocity below 4 $\mathrm{pmol} / \mathrm{mg}$ of protein/min, levels comparable to in vivo dopamine turnover rates (Kuczenski, 1980).

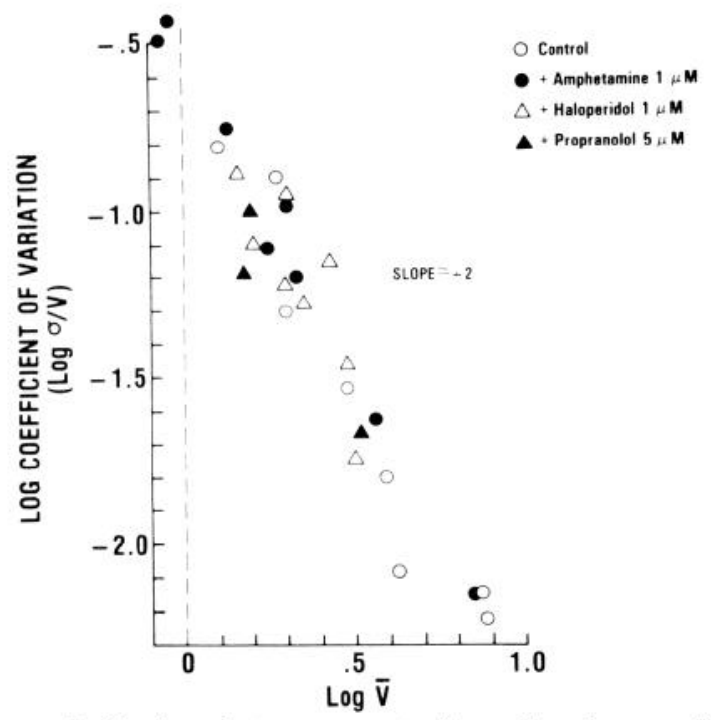

Figure 7. Each point represents the value from a 40-point time series in triplicate. A log-log slope of -2 indicates the quadratic exponential relationship between average velocity and the coefficient of variation which appeared to be uninfluenced by pharmacological ligands present in the supernatant fraction at the concentrations indicated.

pared to controls, neither D-amphetamine, haloperidol, nor propranolol added to the supernatant preparations induced changes in the average amplitude of the fluctuations, which appears log linear with respect to the level of the catalytic velocity, $\bar{V}$.

In contrast to their lack of influence on amplitude, the presence of any of the three drugs in a supernatant preparation results in faster frequencies of variation in TOH activity than controls for a given average amplitude of variation (Fig. 8). Figure 8 also shows that, under control conditions, the higher the coefficient of variation, $\log _{10} \sigma / \bar{V}$, the slower the frequency of variation (lower $D$ ). That might be expected from the changing frequency properties of low and high variance ensembles (Walker, 1931). With the exception of one of the eight control time series, the frequency is log linear with respect to the coefficient of variation, and the suggestion (from Figs. 7 and 8 ) is that, under control conditions, average velocity, average amplitude, and average frequency of variation can be specified when any one of them is known. The symmetries of these properties in some stochastic processes have been used to study chemical reaction kinetics with electrical noise analysis using frequency spectra (Feher and Weissman, 1973). Pilot experiments in which we removed aliquots every minute and incubated for 2 min manifested similar spectra, which suggests that, at

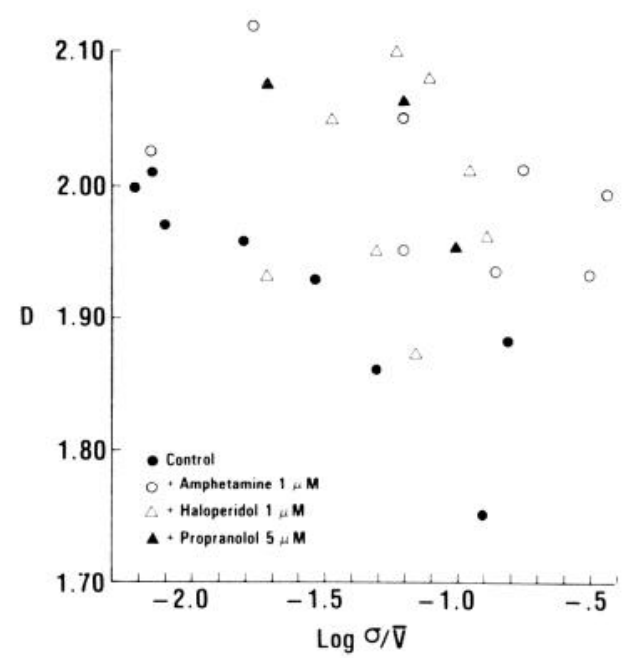

Figure 8. Plotting the $\log$ of the coefficient of variation against $D$ (an exponential index of variational frequency) reveals that, with the exception of one point, the control values manifest a log-linear relationship: the higher the amplitude, the lower the frequency. In contrast, the pharmacological ligands increase the rate of variation, independent of their effects on average amplitude, splitting those two dimensions of a stochastic process which were symmetric under control conditions (Mandell, 1980).

\section{TABLE I}

\section{Averaged relative power spectral distributions}

Each $N$ represents an experiment in which medians of triplicate values from a 40 -point, 80 -min time course were determined in the presence of the ligand as indicated in the text. Spectra of the kinetic variations were normalized and averaged for each condition. The relative power (variance) of each of three dominant frequency peaks $(1 / f$, in minutes) is tabulated as a percentage. The control condition was dominated by the 20 - to 26 -min wavelength; amphetamine tended to increase the power at the intermediate frequency; haloperidol tended to distribute the power more evenly among the frequencies; propranolol elicited regular rhythmicity in the intermediate frequency.

\begin{tabular}{ccccc}
\hline $1 / f$ & $\begin{array}{c}\text { Control } \\
(N=8)\end{array}$ & $\begin{array}{c}\text { Amphetamine } \\
(N=8)\end{array}$ & $\begin{array}{c}\text { Haloperidol } \\
(N=8)\end{array}$ & $\begin{array}{c}\text { Propranolol } \\
(N=3)\end{array}$ \\
\hline $\min$ & & & & \\
$20-26$ & 57.8 & 44.2 & 38.1 & 22.9 \\
$7-8$ & 25.3 & 36.0 & 33.2 & 45.0 \\
$4-5$ & $\underline{16.9}$ & $\underline{19.8}$ & $\underline{28.7}$ & $\underline{102.1}$ \\
& $100 \%$ & $100 \%$ & $100 \%$ & $100 \%$ \\
\hline
\end{tabular}


least within these limits, graining was not determining the frequency peaks.

Table I summarizes averaged spectra from experiments normalized so that the sum of the long, medium, and short wavelengths equals $100 \%$. The presence of pharmacological ligands induced changing patterns in the distribution of power among the three spectral peaks. The longer period was more prominent under control conditions, amphetamine shifted the relative power from the slowest frequency, haloperidol tended to make the power distribution almost equal among the peaks (like "whitening"), and the regular 7- to 8-min peak associated with propranolol is represented by the proportional increase in power in the middle wavelength.

Individual frequency components of fluctuations may indicate independent contributors to variance (Bell, 1960). The persistent presence of multiple spectral frequency peaks, therefore, suggests either the simultaneous presence of three or more kinetic conformations of $\mathrm{TOH}$ or near-harmonic Fourier transformations of the variations around a single kinetic process. Our failure to eliminate the dominant frequency when we filtered with the three-point moving average argues for the former interpretation. We examined the issue further with variously grained substrate curves and the same cofactor concentration used in the time series.

Figure 9 presents a substrate saturation curve at a high cofactor concentration that demonstrates characteristic "substrate inhibition." At this higher level of catalytic velocity, a single hyperbolic function was observed. In contrast, when we observed the pattern of velocity over substrate concentrations increasing in increments of 2 $\mu \mathrm{M}$ with the cofactor concentration at $3 \mu \mathrm{M}$, we found multiple reiterative saturation functions (Fig. 10) of the type first reported by Teipel and Koshland (1969). Figure 11 shows six similar substrate-velocity functions with $\mathrm{BH}_{4}$ at $3 \mu \mathrm{M}$ and tyrosine in increments of 1 or $2 \mu \mathrm{M}$. When considered with the multiple spectral peaks of the time series (Fig. 5 and Table I), these data suggest the presence of either multiple molecular weight forms or multiple quasi-stable states of the same molecular weight form of striatal $\mathrm{TOH}$, each manifesting a different kinetic

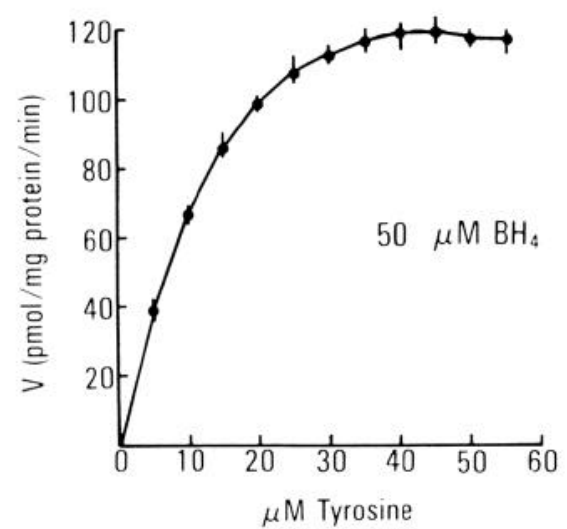

Figure 9. Substrate saturation curve of striatal TOH activity showing the medians and ranges of triplicate values as determined in the presence of $50 \mu \mathrm{M} \mathrm{BH} 4$ cofactor. The function manifests a monotonic, hyperbolic shape with the suggestion of "substrate inhibition" (Shiman and Kaufman, 1970).

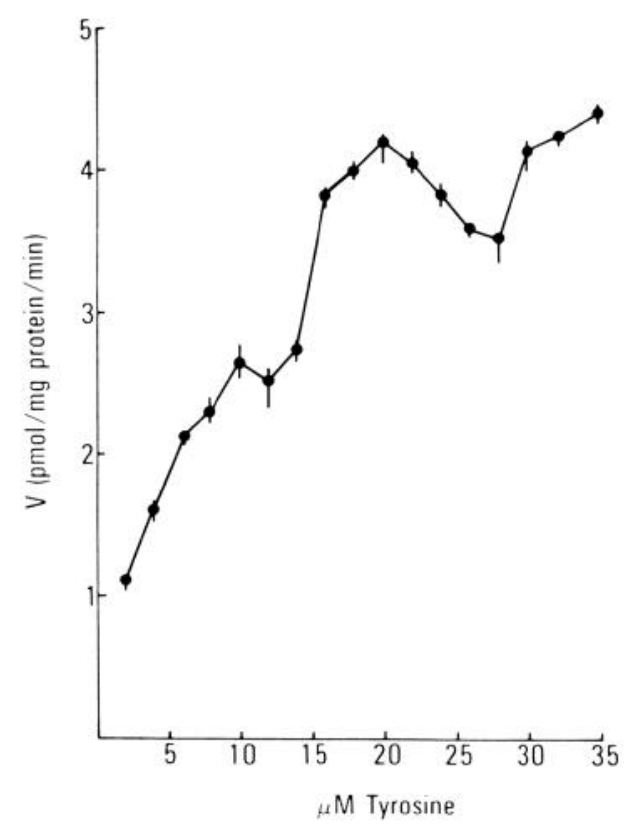

Figure 10. Substrate saturation curve of striatal TOH activity showing the medians and ranges of triplicate values as determined in the presence of $3 \mu \mathrm{M} \mathrm{BH} \mathrm{BH}_{4}$ cofactor. This function manifests multiple reiterative "intermediate saturation plateaus" (Teipel and Koshland, 1969).
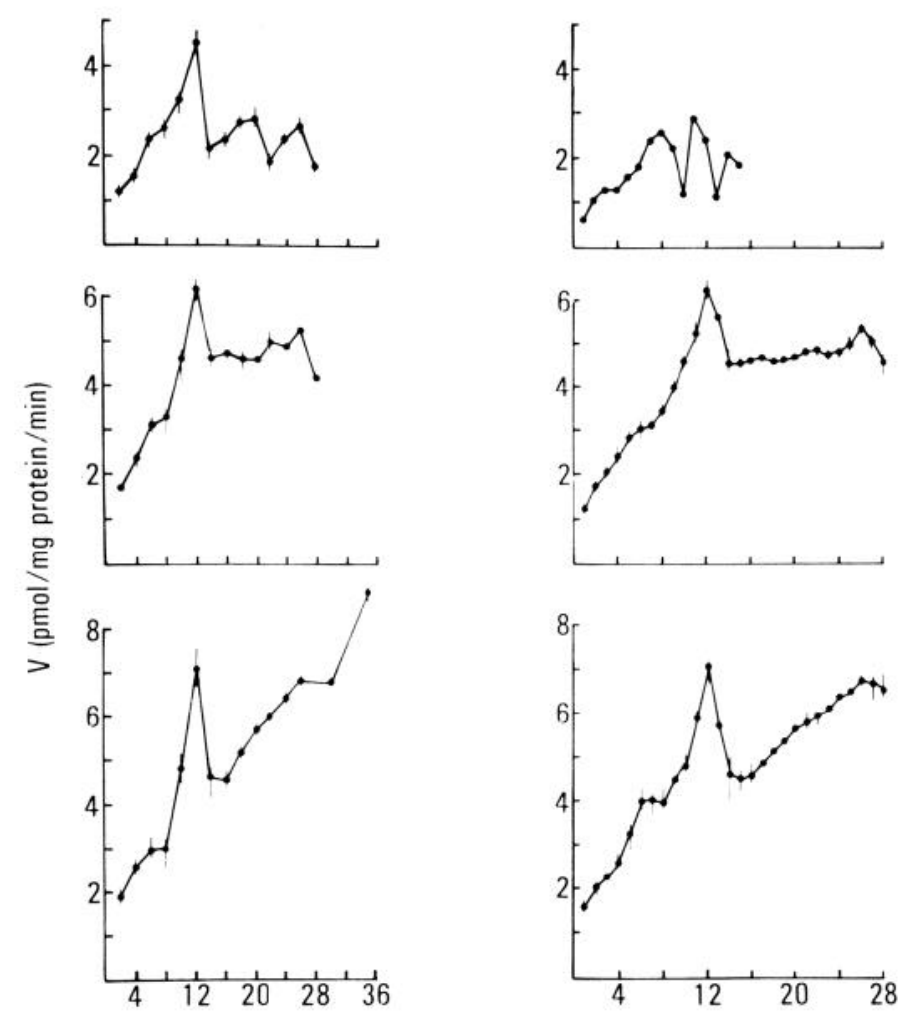

$\mu \mathrm{M}$ Tyrosine

Figure 11. Six additional examples of substrate saturation curves of striatal TOH activity showing the medians and ranges of triplicate values as determined in the presence of $3 \mu \mathrm{M} \mathrm{BH}$ (as in Fig. 10). All six manifest the characteristic multiple kinetic segments and "bumps" seen in the behavior of several regulatory enzymes (Teipel and Koshland, 1969). 
activity with respect to tyrosine concentration and in the frequency domain.

Figure 12 shows the results of column chromatographic separation of enzyme activities and the influence of different molecular weight forms of $\mathrm{TOH}$ on the pattern of substrate saturation functions. Enriched 4- to 6-fold, three distinct fractions of TOH (Musacchio et al., 1973) reflect similar multiplicity of saturation functions and progressively decreasing affinity for tyrosine, independent of their very different molecular weights. The finding is consistent with the occurrence of different kinetic activities within $\mathrm{TOH}$ forms of the same molecular weight or, more generally, with the coexistence of multiple stable states in a globular protein moiety in solution (Woodward and Hilton, 1979; Finney et al., 1980; Leak and Chothia, 1980).

The concurrence of three or more kinetic forms of striatal TOH with different states of activation, as is suggested by the multiple substrate saturation functions in TOH fractions of the same molecular weight, would predict that, whereas high reactant concentrations might promote the symmetrical expression of all forms when studied over time, low reactant concentrations would favor the expression of the kinetic form of the enzyme in the highest activity state.
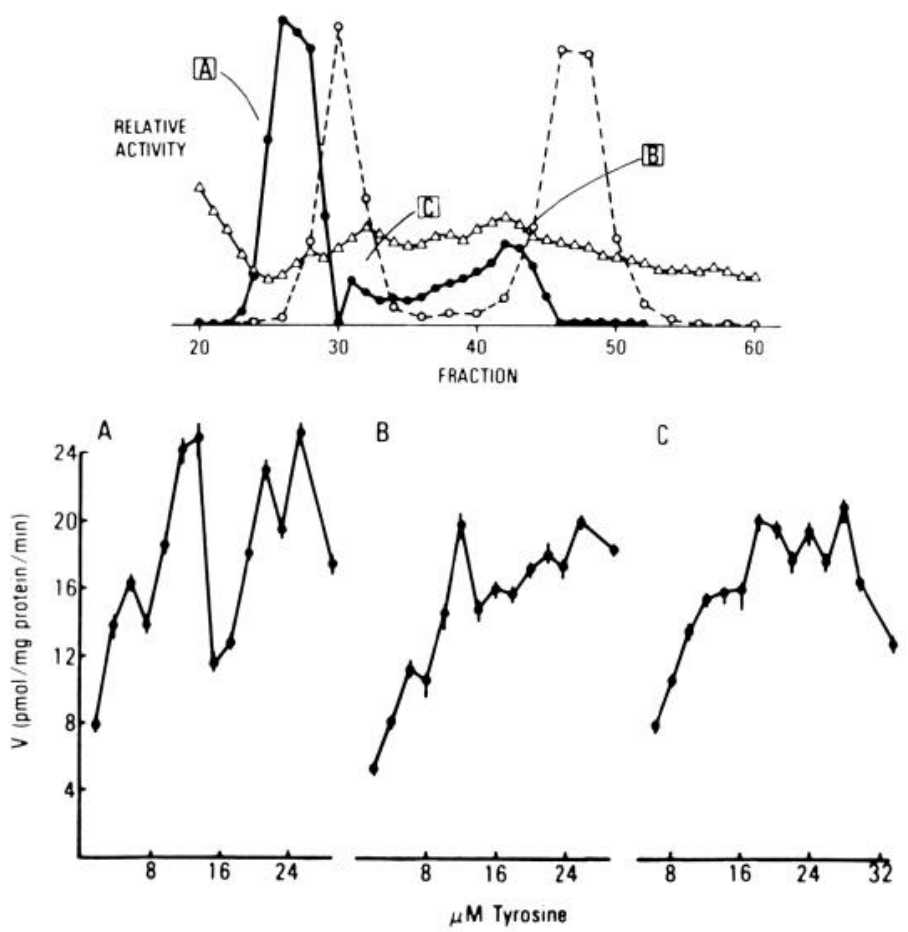

Figure 12. Supernatant fraction of striatal homogenate subjected to column chromatography using an Ultrogel ACA 34 polyacrylamide/agarose polymer with exclusion limits of $M_{\mathrm{r}}=$ 20,000 and 350,000 yielded a 4 - to 5 -fold increase in $\mathrm{TOH}$ activity (-) and three discriminable molecular weight forms $(A, B$, and $C)$ free of quinonoid dihydropteridine reductase activity $\left(\mathrm{O}_{-}-\mathrm{O}^{-}\right.$). Substrate saturation functions determined for each $\mathrm{TOH}$ peak at $3 \mu \mathrm{M} \mathrm{BH}_{4}$ manifested multiple kinetic segments comparable to those seen when the supernatant fraction was used as the enzyme source as in Figures 10 and 11. $\triangle \longrightarrow \triangle$ equals protein determined according to the method of Lowry et al. (1951). See text.
POWER SPECTRAL FREQUENCY DISTRIBUTIONS

AT HIGH AND LOW COFACTOR CONCENTRATIONS

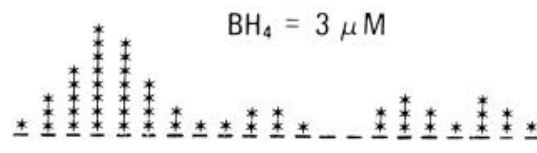

$$
\begin{aligned}
& \mathrm{BH}_{4}=30 \mu \mathrm{M}
\end{aligned}
$$

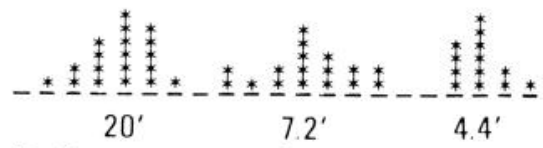

Figure 13. Power spectra from two 40-point, 80-min time courses of $\mathrm{TOH}$ activity as executed in triplicate with the median values used to determine the variational frequency in the time series of deviations from mean velocity at 3 and $30 \mu \mathrm{M}$ $\mathrm{BH}_{4}$ cofactor concentrations. The higher reactant concentration makes the characteristic frequencies of the less activated kinetic conformations more obvious, demonstrating one way in which substrate and/or cofactor concentration may regulate the number and the mix of neurotransmitter concentration frequencies. This finding is consistent with the contemporaneous existence of multiple kinetic forms with varying states of activity across time as suggested by the reiterative saturation functions over increasing substrate in Figures 10 to 12.

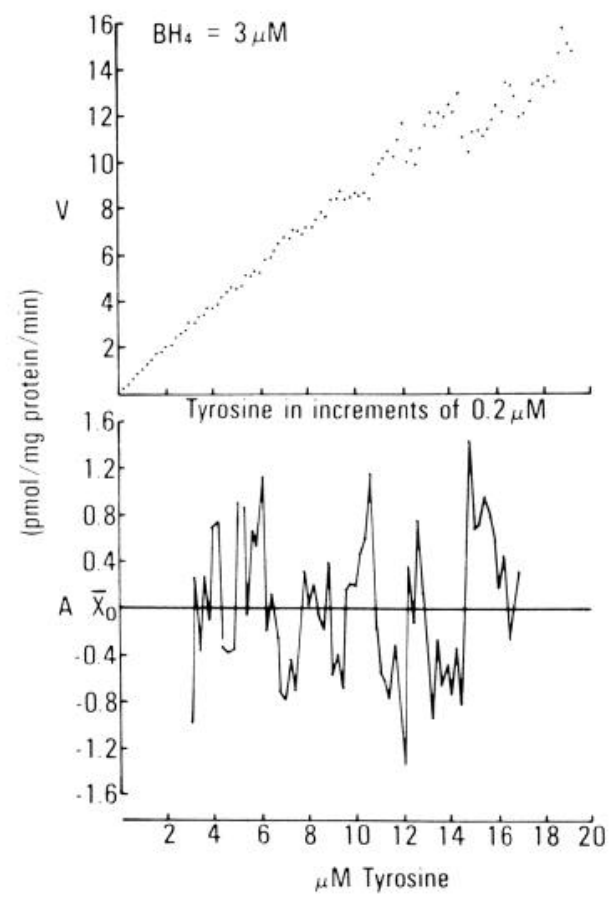

Figure 14. Representative 100-point substrate function of striatal TOH activity determined at a $\mathrm{BH}_{4}$ concentration of 3 $\mu \mathrm{M}$ across $0.2 \mu \mathrm{M}$ increments of tyrosine (upper graph). Assays were performed in triplicate, and the median points are plotted. The fine grain pattern of reiterative kinetic segments is plotted in the lower graph as deviations $(A)$ from the mean velocity determined by linear regression analysis and normalized with respect to velocity and slope. Spectra for these data reveal characteristic peaks in the distribution of variance by frequency in wavelengths of substrate concentration. 
Figure 13 contains frequency (power) spectra from two simultaneous time series (as in Fig. 5 and Table I), illustrating that, at the higher of the two cofactor concentrations ( 30 versus $3 \mu \mathbf{M}$ ), and therefore also at the higher velocity, the power (variance) is more evenly distributed among the three hypothesized kinetic conformations, i.e., among the three prominent frequencies of fluctuation.

Among the multiple kinetic conformations manifested in time series, relative prominence appears to be influenced by drugs with dopaminergic action (Table I). We are proceeding with examination of changes induced by various drugs in the distribution of variance among the stable states of TOH by using more finely grained increments in tyrosine concentration. For example, the top of Figure 14 is a plot of the median points of triplicate determinations over tyrosine increasing in increments of $0.2 \mu \mathrm{M}$ with $\mathrm{BH}_{4}$ at $3 \mu \mathrm{M}$. The bottom of the figure shows the points transformed to deviations $(A s)$ around the linear mean velocity function as determined by regression analysis and normalization. These $A$ values can be treated the way that we treat the deviations from mean velocity in time series, and spectral analysis reveals characteristic peaks in the distribution of variance by frequency in wavelengths of substrate concentration (in this instance, about $3 \mu \mathrm{M}$ (yrosine). As was the case in the time series analysis, amphetamine (Fig. 15) appears to distribute the kinetic power (variance) more evenly over the conformations with differing variational wavelengths in micromolar concentrations of substrate (compare Fig. $5 C$ and Table I).

\section{Discussion}

Rat striatal supernatant fractions that were kept at $4^{\circ} \mathrm{C}$, sampled in triplicate at 2 -min intervals, and assayed

\section{POWER SPECTRAL FREQUENCY DISTRIBUTIONS OF KINETIC VARIATIONS ACROSS INCREASING TYROSINE}

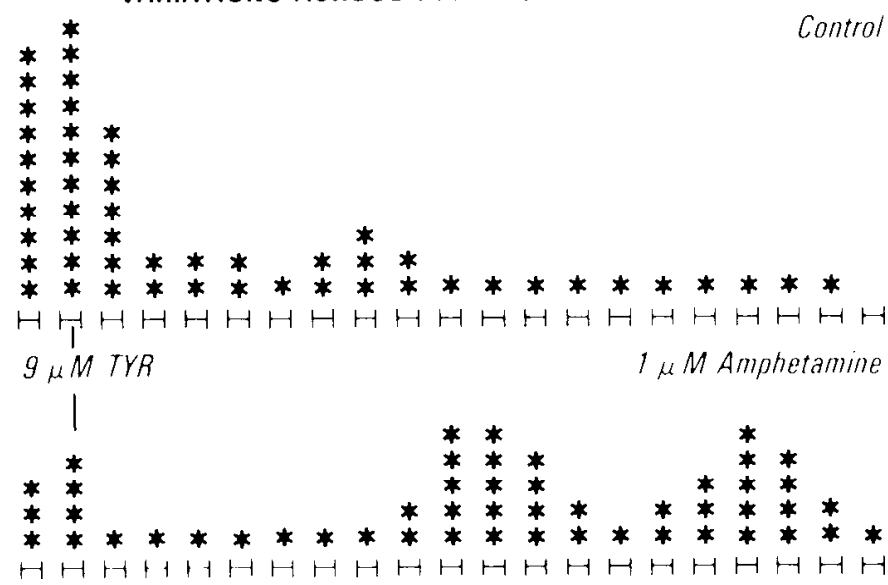

Figure 15. Spectra obtained from two 100-point substrate saturation functions of striatal $\mathrm{TOH}$ activity at a $\mathrm{BH}_{4}$ concentration of $3 \mu \mathrm{M}$ across tyrosine increments of $0.2 \mu \mathrm{M}$ executed in triplicate as for Figure 14. Here, in the presence of $1 \mu \mathrm{M}$ amphetamine, note the more symmetrical distribution of power over the spectral peaks in wavelengths of tyrosine concentration as were seen also in the variational analysis over time with that drug (Fig. $5 C$; Table I). Both findings suggest a drug-induced alteration in the relative time distributions of multiple kinetic conformations with differing activity states. for $1 \mathrm{~min}$ at $37^{\circ} \mathrm{C}$ at the physiological cofactor concentration for $\mathrm{TOH}$ activity manifested characteristic frequencies in variation around the mean velocity, $\bar{V}$. The average amplitude of the variations, $\sigma$, manifested a quadratic relationship to $\bar{V}$ below $4 \mathrm{pmol} / \mathrm{mg}$ of protein/ min and a zero slope above that velocity; $\sigma$ appeared unchanged (beyond the change associated with the mean velocity) by higher temperatures, D-amphetamine, haloperidol, or propranolol.

In contrast, the frequency of variations around the mean velocity slope, as reflected by power spectra, autocorrelation functions, and the dimensional exponent, $D$, was increased by higher temperature and by micromolar concentrations of the same three drugs. There is a suggestion that each drug may have induced a characteristic distribution of power among the frequencies. Column chromatographic separation of three dominant peaks of 'TOH activity and subsequent substrate-velocity plots with $\mathrm{BH}_{4}$ at $3 \mu \mathrm{M}$ revealed multiple, reiterative saturation functions in all three fractions. Along with the multiple spectral peaks of kinetic variations in time, that suggested the simultaneous presence of multiple kinetic conformations (oscillators), in different states of activation, manifesting characteristic product concentration frequencies. Low tyrosine concentrations appeared to select one and high tyrosine concentrations three kinetic conformations of TOH. A pharmacological influence on the relative distribution of multiple kinetic forms of TOH could be seen in the pattern of kinetic variation over substrate as well as over time.

Cell-free and membrane-free oscillatory behavior in enzyme activities has been observed in photosynthetic, oxidative, glycolytic, and microsomal systems, including those in the brain, in mammals and other species (Hess and Boiteux, 1971; Goldbeter and Kaplan, 1976). At $37^{\circ} \mathrm{C}$, characteristic periods range from 1 to $5 \mathrm{~min}$, and the mechanistic explanation usually has been kinetic, proposing that interactions of critical reactant concentrations and affinities of an allosteric, cooperative enzyme system lead to the evolution of quasi-periodic metabolite and free enzyme concentrations that can be described with a set of ordinary differential equations (Chance et al., 1964; Hess et al., 1978).

A contrasting, non-kinetic view of time-dependent macromolecular structural and functional instability has arisen from recent research in protein physics. Such methods as fluorescence quenching and polarization, phosphorescence, $\mathbf{x}$-ray scattering, nuclear magnetic resonance, pulse relaxation, dielectric spectra, temperature jump, and tritium exchange have revealed the globular protein in solution as a fluid, dynamic structure with multiple quasi-stable conformations having low energy barriers between them (as little as $5 \mathrm{~kJ} / \mathrm{mol}$ ). Autonomous conformational fluctuations can be observed across a range of time scales, from picoseconds to hours (Careri et al., 1979; Woodward and Hilton, 1979). Conformational (fold) instability correlates well with the ratio of charged (destabilizing) to hydrophobic (stabilizing) amino acid residues across protein types (Williams, 1979), and thermodynamic calculations have demonstrated the magnitude of Gibbs free energy fluctuations in a globular protein in solution at $25^{\circ} \mathrm{C}$ to be as large as the mean enthalpy changes accompanying protein denaturation 
(Cooper, 1976). Thus, an enzyme protein can be viewed as a fluctuating structure with the rate of the process depending on factors that influence its structural stability.

Because the temperature-sensitive quasi-periodicities of about $26,7.5$, and $5 \mathrm{~min}\left(\right.$ at $4^{\circ} \mathrm{C}$ ) are longer than our 1-min assay incubations, our present findings cannot be accounted for in terms of sequential kinetic interactions. More likely explanations for the changes observed in kinetic variational frequency would be conformational influences by ligands and drugs on solvent accessibility, protein surfaces, and folding-the forces stabilizing macromolecular structure at the water-protein interface (Finney et al., 1980; Leak and Chothia, 1980), influencing the relative time distribution among multiple quasi-stable states characteristic of globular proteins in solution.

Tanford (1968) has reported a similar sequence of multiple zero slope plateaus over the course of the denaturation of a globular protein with increasing salt concentration. Teipel and Koshland (1969) also reported a similar sequence when they examined the saturation kinetics across reactant and ligand concentrations of a number of regulatory enzymes. Both the multiple spectral peaks of the kinetic variations in time and the multiple substrate saturation plateaus across increasing substrate in our studies are consistent with their findings and support the possibility of concurrent multiple conformations of the globular protein in solution, each with a definable activity state and a characteristic variational frequency in time.

The insensitivity of the average amplitude of the kinetic variations in striatal TOH activity to temperature and pharmacological ligands and the asymptotic, exponential dependence of that value on the level of catalytic velocity are consistent with the statistical characteristics of variance when high and low levels of a stochastic process are compared (Hogg and Craig, 1970). Enzyme reaction rates determined under equilibrium conditions, with high reactant concentrations, manifest the smaller variance-to-mean ratio of a binomial distribution of intermediate probability, which is stable beyond a critical range of catalytic velocities (seen in Fig. 6 above $4 \mathrm{pmol} /$ $\mathrm{mg}$ of protein/min). Lower $\mathrm{BH}_{4}$ concentrations and the resulting low catalytic velocities consonant with endogenous biosynthesis rates reflect longer (and more variable) intervals between cofactor "hits" on the enzyme protein. This kind of a stochastic process, a binomial distribution of low probability, leads to a Poisson distribution in which the amplitude of the variance approaches the mean: the lower the mean level of a time-dependent process, the larger the available interval of time in which to vary. Therefore, it is our hypothesis that whereas frequency of variations reflects the time dependence of changing macromolecular stability and an accompanying change in the relative prominence of multiple kinetic conformations, their amplitude is a function of the distributional characteristics of a non-equilibrium Poisson process associated with low (physiological) reactant concentrations. Recently we have observed kinetic variations with similar properties in pure bovine pancreatic ribonuclease $\mathrm{A}, \mathrm{B}$, and $\mathrm{S}$, whose frequency, but not amplitude, was influenced by pharmacological ligands ( $S$. Knapp and A. J. Mandell, manuscript in preparation).
The frequency of variations has been a dimension of measurement in studies of membrane ion conductances (Nelson et al., 1980), of the interspike intervals of spontaneous neuronal discharge (Moore et al., 1966), and of the electroencephalograph (Gehrmann and Killam, 1978). This "second-order" variation (Prigogine, 1965) may prove useful also in studies of the entropy in brain chemical systems. In addition, chemical wave processes may play a role in neurobiological function. Perlow et al. (1979) have reported that motor abnormalities produced by destruction of the nigrostriatal dopamine system are ameliorated partially by grafts of brain dopaminergic as well as adrenal medullary tissue. A property of TOH function that would maintain coherence over space and time in dopamine synthesis wave processes without axonal or synaptic mechanisms suggests a possible explanation for such findings.

Several possibilities arise if kinetic conformers function as statistical oscillators. The multiple spectral peaks may represent either a single average frequency with harmonic side bands or multiple oscillators with differing angular frequencies leading to additional "beat" frequencies. Reiterative nonlinearities in the kinetic functions may portray phase relations among the participating oscillators with stationary constructive and destructive interference patterns. Variational rate may be an alternative description for an affinity constant expressed as a probability density, transformed into a frequency via a Fourier characteristic function (spectrum) or a characteristic exponent $(D)$. These and related possibilities are currently being explored.

\section{References}

Bell, D. A. (1960) Electrical Noise, pp. 1-36, Van Nostrand, New York.

Bendat, J. S., and A. G. Piersol (1971) Random Data: Analysis and Measurement Procedures, pp. 170-213, Wiley-Interscience, New York.

Blackman, R. B., and J. W. Tukey (1958) The Measurement of Power Spectra, pp. 57-65, Dover, New York.

Bullard, W. P., P. B. Guthrie, P. V. Russo, and A. J. Mandell (1978) Regional and subcellular distribution and some factors in the regulation of reduced pterins in rat brain. J. Pharmacol. Exp. Ther. 206: 4-20.

Careri, G., P. Fasella, and E. Gratton (1979) Enzyme dynamics: The statistical physics approach. Annu. Rev. Biophys. Bioeng. 8: 69-97.

Chance, B., A. Ghosh, J. J. Higgins, and P. K. Maitra (1964) Cyclic and oscillatory responses of metabolic pathways involving chemical feedback and their computer representations. Ann. N. Y. Acad. Sci. 115: 1010-1024.

Chatfield, C. (1975) The Analysis of Time Series: Theory and Practice, pp. 12-31, 110-168, Chapman and Hall, London.

Cooper, A. (1976) Thermodynamic fluctuations in protein molecules. Proc. Natl. Acad. Sci. U. S. A. 73: 2740-2741.

Feher, G., and M. Weissman (1973) Fluctuation spectroscopy: Determination of chemical reaction kinetics from the frequency spectrum of fluctuations. Proc. Natl. Acad. Sci. U. S. A. 70: 870-875.

Finney, J. L., B. J. Gellatly, J. C. Golton, and J. Goodfellow (1980) Solvent effects and polar interactions in the structural stability and dynamics of globular proteins. In Protein and Nucleoproteins: Structure, Dynamics, and Assembly, pp. 131-142, Rockefeller University Press, New York.

Gehrmann, J. E., and K. F. Killan (1978) Studies of central 
functional equivalents. I. Time-varying distribution of power in discrete frequency bands of the EEG as a function of drug exposure. Neuropharmacology 17: 747-759.

Geyer, M. A., A. Puerto, W. J. Dawsey, S. Knapp, W. P. Bullard, and A. J. Mandell (1976) Histologic and enzymatic studies of the mesolimbic and mesostriatal serotonergic pathways. Brain Res. 106: 241-256.

Goldbeter, A., and S. R. Kaplan (1976) Oscillatory enzymes. Annu. Rev. Biophys. Bioeng. 5: 449-475.

Hess, B., and A. Boiteux (1971) Oscillatory phenomena in biochemistry. Annu. Rev. Biochem. 40: 237-258.

Hess, B., A. Goldbeter, and R. Lefeuer (1978) Temporal, spatial, and functional order in regulated biochemical and cellular systems. Adv. Chem. Phys. 38: 363-413.

Hogg, R. V., and A. T. Craig (1970) Introduction to Mathematical Statistics, pp. 86-111, Macmillan, London.

Knapp, S., A. J. Mandell, and W. P. Bullard (1975) Calcium activation of brain tryptophan hydroxylase. Life Sci. 16: 1583-1594.

Kuczenski, R. (1973) Rat brain tyrosine hydroxylase: Activation by limited tryptic proteolysis. J. Biol. Chem. 248: 2261-2265.

Kuczenski, R. (1980) Amphetamine-haloperidol interactions on striatal and mesolimbic tyrosine hydroxylase activity and dopamine metabolism. J. Pharmacol. Exp. Ther. 215: 135142.

Leak, A. M., and C. Chothia (1980) Solvent accessibility, protein surfaces, and protein folding. In Protein and Nucleoproteins: Structure, Dynamics, and Assembly, pp. 143-152, Rockefeller University Press, New York.

Lowry, O. H., N. J. Rosebrough, A. L. Farr, and R. J. Randall (1951) Protein measurement with the Folin phenol reagent. J. Biol. Chem. 193: 265-275.

Mandelbrot, B. B. (1977) Fractals: Form, Chance, and Dimension, pp. 27-80, W. H. Freeman, San Francisco.

Mandell, A. J. (1978) Redundant mechanisms regulating brain tyrosine and tryptophan hydroxylases. Annu. Rev. Pharmacol. Toxicol. 18: 461-493.

Mandell, A. J. (1980) Vertical integration of levels of brain function through parametric symmetries within self-similar stochastic fields. In Information Processing in the Nervous System, H. M. Pinsker and W. D. Willis, Jr., eds., pp. 177197, Raven Press, New York.

Mandell, A. J. (1981) Statistical stability in random brain systems: Possible implications for polydrug abuse in the borderline syndrome. Adv. Substance Abuse 2: in press.

Mandell, A. J., W. P. Bullard, J. B. Yellin, and P. V. Russo (1980) The influence of $\mathrm{D}$-amphetamine on rat brain striatal reduced biopterin concentration. J. Pharmacol. Exp. Ther. 213: 569-574.

Mandell, A. J., K. D. Stewart, and P. V. Russo (1981) The Sunday syndrome: From kinetics to altered consciousness. Fed. Proc., in press.
Moore, G. P., D. H. Perkel, and J. P. Segundo (1966) Statistical analysis of functional interpretation of neuronal spike data. Annu. Rev. Physiol. 28: 493-522.

Musacchio, J. M., C. A. McQueen, and G. L. Craviso (1973) Tyrosine hydroxylase: Subcellular distribution and molecular and kinetic characteristics of the different enzyme forms. In New Concepts in Neurotransmitter Regulation, A. J. Mandell, ed., pp. 69-88, Plenum Press, New York.

Nagatsu, T., M. Levitt, and S. Udenfriend (1964) A rapid and simple radioassay for tyrosine hydroxylase. Anal. Biochem. 9: $122-126$.

Nelson, N., R. Anholt, J. Lindstrom, and M. Montal (1980) Reconstitution of purified acetylcholine receptors with functional ion channels in planar lipid bilayers. Proc. Natl. Acad. Sci. U. S. A. 77: 3057-3061.

Nielsen, K. H., V. Simonsen, and K. E. Lind (1969) Dihydropteridine reductase: A method for the measurement of activity and investigations of the specificity for NADH and NADPH. Eur. J. Biochem. 9: 497-502.

Perlow, M. J., W. J. Freed, B. J. Hoffer, A. Seiger, L. Olson, and R. J. Wyatt (1979) Brain grafts reduce motor abnormalities produced by destruction of nigrostriatal dopamine system. Science 204: 643-647.

Prigogine, I. (1965) Non-Equilibrium Thermodynamics, Variational Techniques, and Stability, University of Chicago Press, Chicago.

Shiman, R., and S. Kaufman (1970) Tyrosine hydroxylase. Methods Enzymol. 17A: 609-615.

Slutzky, E. (1937) The summation of random causes as the source of cyclic processes. Econometrica 5: 105-146.

Stevens, C. F. (1977) Study of membrane permeability changes by fluctuation analysis. Nature 270: 391-396.

Tanford, C. (1968) Protein denaturation, Part B. The transition from native to denatured state. Adv. Protein Chem. 23: 218282.

Teipcl, J., and D. E. Koshland (1969) The significance of intermediary plateau regions in enzyme saturation curves. Biochemistry 8: 4656-4663.

Walker, G. (1931) On periodicity in series of related terms. Proc. R. Soc. Lond. (A) 131: 518-532.

Webber, S., and J. M. Whitely (1978) Pyridine nucleotide interaction with rat liver dihydropteridine reductase. J. Biol. Chem. 253: 6724-6729.

Williams, R. J. P. (1979) The conformational properties of proteins in solution. Biol. Rev. 54: 389-437.

Woodward, C. K., and B. D. Hilton (1979) Hydrogen exchange kinetics and internal motions in proteins and nucleic acids. Annu. Rev. Biophys. Bioeng. 8: 99-127.

Yellin, J. B., P. V. Russo, and A. J. Mandell (1979) Cell-free oscillations in striatal dopamine synthesis: Arrest by micromolar lithium. Fed. Proc. Abstr. 2742. 